\title{
Signature of mass supply to quiet coronal loops
}

\author{
H. Tian ${ }^{1,2}$, C.-Y. Tu ${ }^{1,2}$, E. Marsch ${ }^{1}$, J.-S. He ${ }^{2}$, and G.-Q. Zhou ${ }^{2}$ \\ 1 Max-Planck-Institut für Sonnensystemforschung, Katlenburg-Lindau, Germany \\ e-mail: tianhui924@163.com, marsch@mps.mpg.de \\ 2 Department of Geophysics, Peking University, Beijing, PR China \\ e-mail: chuanyitu@pku.edu.cn \\ Received 9 October 2007 / Accepted 30 November 2007
}

\begin{abstract}
Aims. The physical implication of large blue shift of Ne VIII in the quiet Sun region is investigated in this paper.

Methods. We compare the significant Ne VIII blue shifts, which are visible as large blue patches on the Doppler-shift map of a middlelatitude quiet-Sun region observed by SUMER, with the coronal magnetic-field structures as reconstructed from a simultaneous photospheric magnetogram by means of a force-free-field extrapolation.

Results. We show for the first time that coronal funnels also exist in the quiet Sun. The region studied contains several small funnels that originate from network lanes, expand with height and finally merge into a single wide open-field region. However, the large blue shifts of the Ne VIII line are not generally associated with funnels. A comparison between the projections of coronal loops onto the solar $x-y$-plane and the Ne VIII dopplergram indicates that there are some loops that reveal large Ne VIII blue shifts in both legs, and some loops with upflow in one and downflow in the other leg.

Conclusions. Our results suggest that strong plasma outflow, which can be traced by large Ne vIII blue shift, is not necessarily associated with the solar wind originating in coronal funnels but appears to be a signature of mass supply to coronal loops. Under the assumption that the measured Doppler shift of the Ne VIII line represents the real outflow velocity of the neon ions being markers of the proton flow, we estimate the mass supply rate to coronal loops to be about $10^{34} \mathrm{~s}^{-1}$.
\end{abstract}

Key words. Sun: corona - Sun: transition region - Sun: UV radiation - Sun: magnetic fields - Sun: solar wind

\section{Introduction}

Information on the physics of the solar transition region and corona can be extracted from the solar ultraviolet emission. Since the plasma is optically thin for most of the emission lines, their profiles indicate the physical conditions prevailing in the emission regions. For instance, the line-of-sight velocity of the plasma can simply be inferred by using the Doppler shift formula $v_{\text {los }}=c\left(\lambda-\lambda_{0}\right) / \lambda_{0}$, where $\lambda_{0}$ is the wavelength at rest, $\lambda$ is the observed wavelength of a line, and $c$ is the speed of light in vacuum.

Owing to the high spectral resolution of the SUMER (Solar Ultraviolet Measurements of Emitted Radiation) instrument onboard SOHO (Solar and Heliospheric Observatory), the Doppler shifts of spectral lines can be measured with an accuracy of about 1-2 $\mathrm{km} \mathrm{s}^{-1}$ (Brekke et al. 1997; Hassler et al. 1999; Peter \& Judge 1999; Wilhelm et al. 2000). Hundreds of emission lines in the SUMER spectral window have been identified by Curdt et al. (2004). The Ne VIII ( $\lambda 770)$ resonance line $\left(2 s^{2} S_{1 / 2}-2 p^{2} P_{3 / 2}\right)$ is of particular interest for the study of the upper solar transition region. Since Dammasch et al. (1999) derived a very accurate rest wavelength of $(770.428 \pm 0.003) \AA$ for this line, the Ne VIII Doppler shift can be precisely determined and thus has been intensively studied in the recent past.

Generally, the Ne VIII line was used to study the origin of the solar wind, and the blue shift of this line is considered to be a signature of solar-wind outflow. Sizable patches of large blue shift were found in the Ne VIII dopplergrams in coronal holes (Peter 1999; Stucki et al. 2000; Wilhelm et al. 2000; Aiouaz et al. 2005); they tend to be larger in the darker regions of coronal holes (Xia et al. 2003). Hassler et al. (1999) studied the relationship between Doppler shift and chromospheric network and found that a larger blue shift is closely associated with the underlying chromospheric network. Tu et al. (2005a) then found that the patches of large Ne VIII blue shift are connected with coronal funnels, which were reconstructed by the method of force-freefield extrapolation.

In the quiet Sun, large Ne VIII blue shifts were also found in the network lanes and considered to indicate the sources of the solar wind (Hassler et al. 1999). However, such different mechanisms as solar wind outflows, spicules, siphon flows through loops, nano-flares and explosive events all can cause Doppler shifts of transition region lines (Peter \& Judge 1999). Strong adjacent up and down flows have been detected in magnetically active regions associated with sunspots (Marsch et al. 2004). We must therefore carefully check whether or not the surrounding magnetic environment is suitable for a certain mechanism to take effect on the Sun. To this end, He et al. (2007) reconstructed the coronal magnetic field in the quiet Sun by the method of force-free-field extrapolation, and they found that most of the sites with Ne VIII blue shift were not located in regions with an open magnetic field. Thus they claimed that these sites may not be sources of the solar wind. They also found that, one dark area on the $\mathrm{Fe}(\lambda 195)$ radiance map seemed to be connected with open field lines, and conjectured that it could be a source of the solar wind.

In this paper, we will present new results on the relationship between the coronal magnetic field and the Ne VIII blue shift. Since open and closed field lines can reach different heights, we will trace the magnetic field lines going up and down, while 
starting at every grid point uniformly distributed on a plane at different heights in our calculation box, in order to illustrate the two basic kinds of coronal magnetic structures - funnels and loops - and to establish their association with the Ne VIII blue shift.

\section{Data analysis and results}

On 22 September 1996, SUMER observed a middle-latitude quiet-Sun region from 00:40 to 08:15 UTC. There were three main emission lines, Si II $(153.3 \mathrm{~nm})$, C IV $(154.8 \mathrm{~nm})$, and $\mathrm{Ne}$ VIII $(77.0 \mathrm{~nm})$ in the selected spectral window. In this paper, we will concentrate on the Doppler shift of Ne VIII. This data set has been intensively studied before by Dammasch et al. (1999), Hassler et al. (1999), Peter (2000), Gontikakis et al. (2003), Tu et al. (2005b), and He et al. (2007). More details about the observations can be found in these papers.

The standard SUMER procedures for correcting and calibrating the data were applied. We obtained the Ne VIII dopplergram by using the same method as described in Tu et al. (2005b). We used the magnetogram taken by the Michelson Doppler Imager (MDI) on SOHO on the same day at 01:39 UTC. The pixel size of MDI is about $2^{\prime \prime}$. The correction of the magnetogram and the coalignment of the magnetogram with the SUMER images were carried out by applying the same method described in Tu et al. (2005b), and therefore are not addressed here.

In our present study we used the force-free-field extrapolation method as proposed by Seehafer (1978) to extrapolate the photospheric magnetic field to $80 \mathrm{Mm}$ above the photosphere. Thus, the size of the calculation box is $442^{\prime \prime} \times 259^{\prime \prime} \times 80 \mathrm{Mm}$. Those field lines reaching heigher than $80 \mathrm{Mm}$ are defined as open, otherwise they are defined as closed. The description of the extrapolation process, as well as of the suitability of this method for our study, can be found in Tu et al. (2005b).

We traced the magnetic field lines going up and down in the corona, while starting at every grid point uniformly distributed over a plane located at $20 \mathrm{Mm}$ in our calculation box. Figure 1 shows the distribution of the foot points of these magnetic field lines. The lanes of the chromospheric network were extracted from a Si II intensity image and are reproduced from the paper by Hassler et al. (1999). It is clear that almost all of the foot points of the field lines, whether being closed or open, originate from network lanes and tend to cluster in the network. Since these dots represent the foot points of field lines reaching higher than $20 \mathrm{Mm}$, the red and blue clusters of dots can be considered as the bottom regions of open funnels and large closed loops, respectively.

He et al. (2007) found that in a cross-section plane located at a height of $25 \mathrm{Mm}$, the pattern of open field lines intersecting that plane is consistent with the dark pattern of low radiance in the image of Fe XII ( $\lambda 195)$. They claimed that this dark region might be a source of solar wind. Since in coronal holes the solar wind appears to originate from funnels, it is natural for us to raise the question whether or not we can also identify funnels on the basis of the observations of this region. We followed this idea and reconstructed, for the first time, a coronal funnel structure in the quiet Sun based on the extrapolation from the photospheric magnetic field. The results are illustrated in Fig. 2.

Each small funnel below $20 \mathrm{Mm}$ originates from a cluster of foot points of open field lines. These small funnels expand with height and merge into a single open field region above $20 \mathrm{Mm}$. The total area of the cross section at $20 \mathrm{Mm}$ of all the small funnels together is close to that of the resulting merger funnel.

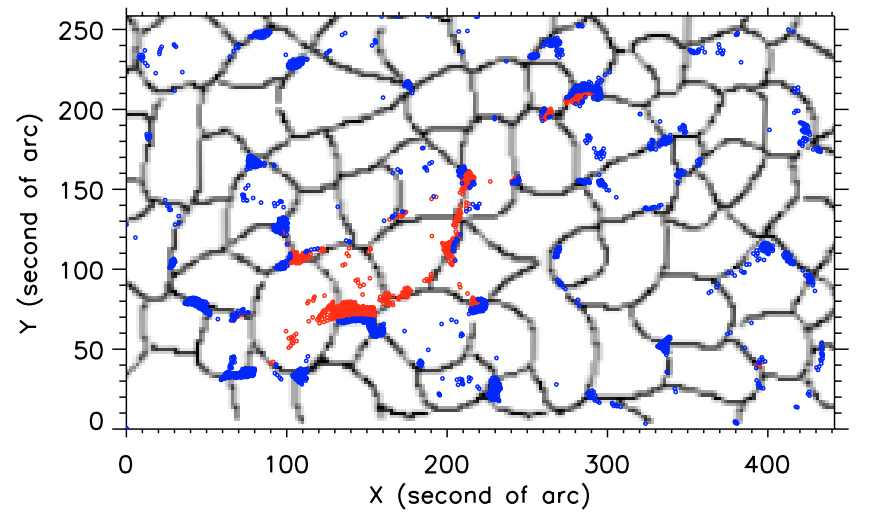

Fig. 1. Foot points of magnetic field lines higher than $20 \mathrm{Mm}$ are shown overlaid on the pattern of the chromospheric network, which was extracted from a Si II intensity image and is reproduced from the paper by Hassler et al. (1999). The red and blue dots denote the foot points of open and closed field lines, respectively.

From Fig. 1 we can also see some scattered red dots, which are the foot points of the black field lines in Fig. 2. These lines are also open and go into the funnel merging region, but they do not originate in any of the small funnels. Aiouaz \& Rast (2006) predicted that open flux in the network can be redistributed into the internetwork region, allowing some of the internetwork field to remain open in the corona. The "isolated" open field lines in our result seem to originate from the internetwork region, which confirms this prediction.

In their study, He et al. (2007) found that most of the sites showing plasma outflow were not located in regions with an open magnetic field. From inspection of Fig. 2 we also find that most of the funnel bottoms do not coincide with patches of strong Ne VIII blue shift. Only one small funnel reveals considerable blue shift at its bottom (around the coordinates $x=220^{\prime \prime}$ and $y=75^{\prime \prime}$ in the $x-y$ plane), while small blue shifts or even red shifts are associated with the other small funnels.

As we have now established that large blue shifts in the quiet Sun mostly do not coincide with funnel bottoms, it seems natural to consider whether there is some connection between large blue shifts and coronal loops. Since they are thought to reside mainly in the lower part of the corona, we traced the magnetic field lines going up and down, while starting at every grid point uniformly distributed in a lower plane at $4 \mathrm{Mm}$ in our calculation box. We then projected the extrapolated magnetic loops reaching higher than $4 \mathrm{Mm}$ onto the tangent $x-y$-plane, where the coordinate $x$ refers to east-west and y south-north direction.

Figure 3 shows the results of our correlation study. The contours of the magnetic field strength coincide well with patches of large blue shift of Ne VIII, a similar result to that previously found by Tu et al. (2005b). However, there clearly are some cases where both legs of the loop correspond to large blue shift of Ne VIII. But there are also some loops with upflow and downflow in their different legs. It appears as if the magnetic fields in or around both legs of the loop are strong and comparable, then the blue shift tends to be strong in both legs. In contrast, if the magnetic fields are not comparable, one finds blue shift in one leg and red shift in the other.

We plotted the Ne VIII Doppler shift and the line-of-sight component of the observed photospheric magnetic field strength along different cuts, which are shown as yellow lines in Fig. 3. The extrapolated magnetic field lines are projected on the vertical plane corresponding to each of the four cuts through the 

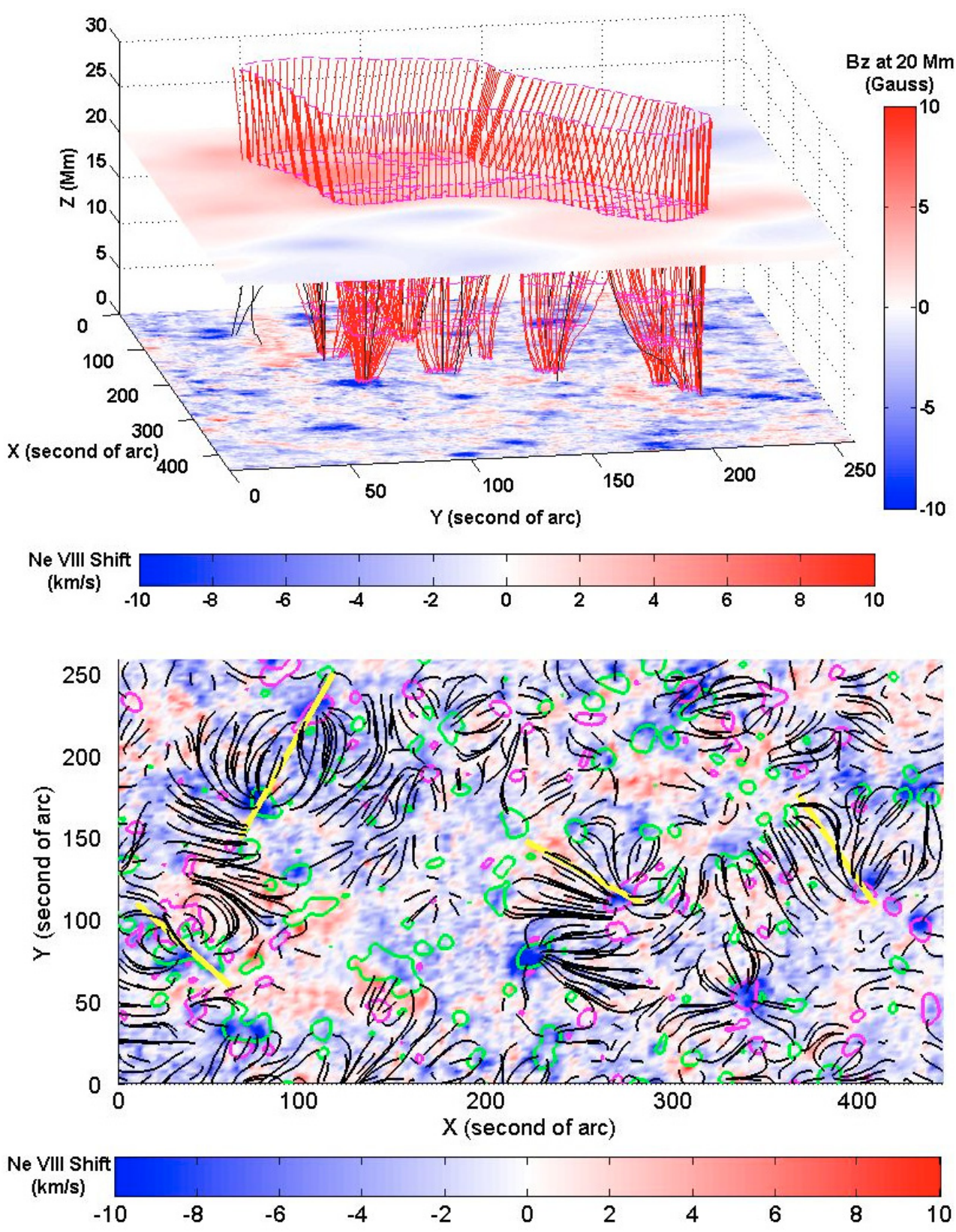

Fig. 2. Magnetic funnels in a quiet sun region. The red lines are field lines originating from the funnel boundary, and the black ones are open field lines outside small funnels. The Ne VIII Doppler shift image is placed at zero megameter, and the color coding is given at the bottom. The positive and negative values represent red shifts and blue shifts, respectively. The map of the vertical component of the extrapolated magnetic field at $20 \mathrm{Mm}$ is placed at the height of $20 \mathrm{Mm}$, and the color coding is given on the right-hand bar.
Fig. 3. Projections of the extrapolated magnetic loops reaching higher than $4 \mathrm{Mm}$ onto the $x-y$-plane, together with the map of the Ne VIII Doppler shift. Regions with positive and negative magnetic flux density are outlined in green and pink color, with the contour level of $15 \mathrm{G}$ and $-15 \mathrm{G}$, respectively. The four yellow lines correspond to the four horizontal axes in Fig. 4. extrapolation box. Figure 4 presents the result. For the two cases shown in the top panel, it is clear that both legs of the big loop correspond to large Ne VIII blue shift, and there are strong magnetic fields (more than 40 Gauss) in or around both legs. For the two cases shown in the bottom panel, only one leg of the big loop corresponds to a large Ne VIII blue shift, whereas the other one corresponds to a red shift. Also, the magnetic field is asymmetric and different in the two legs; it tends to be much stronger in or around the leg where the Ne VIII blue shift occurs.

\section{Conclusion and discussion}

Stimulated by previous work of He et al. (2007), who already pointed out that most locations with strong blue shifts cannot be identified with the foot points of open field lines and hence may not be source regions of the solar wind in the quiet Sun, we further studied in depth the relationship between strong Ne VIII blue shift and the coronal magnetic field structure. We found that there is a good coincidence of most locations of strong blue shifts with legs of large coronal loops.

There are also some loops with upflow and downflow in their different legs, which can be explained by the so-called siphon flow, where the gas flow is possibly driven by a strong asymmetric heating in the loop (McClymont \& Craig 1987; Mariska 1988; Spadaro et al. 1991). This type of flow may cause blue shift in one leg and red shift in the other. The observed asymmetry of the magnetic field strength in the two loop legs seems to favor such uneven heating. However, shifts with opposite directions in the two legs may also be caused by different mechanisms, e.g., with the red shift resulting from any one of the previously suggested mechanisms (Brekke et al. 1997; Peter \& Judge 1999), and with the prominent blue shift being caused by plasma entering into the loop leg from outside.

Some of the quiet-Sun-area loops observed here have significant outflows in both legs, which according to our knowledge, is reported here for the first time. We suggest that these outflows are caused by plasma entering into the loop legs from outside by 


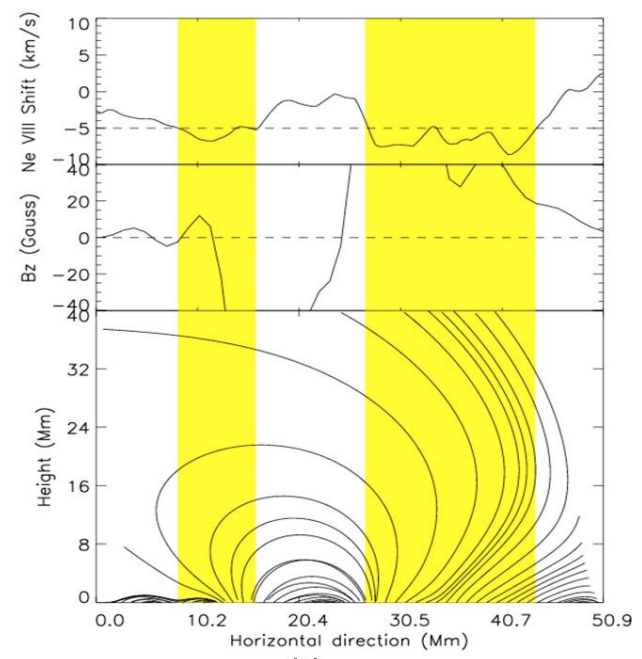

(a)

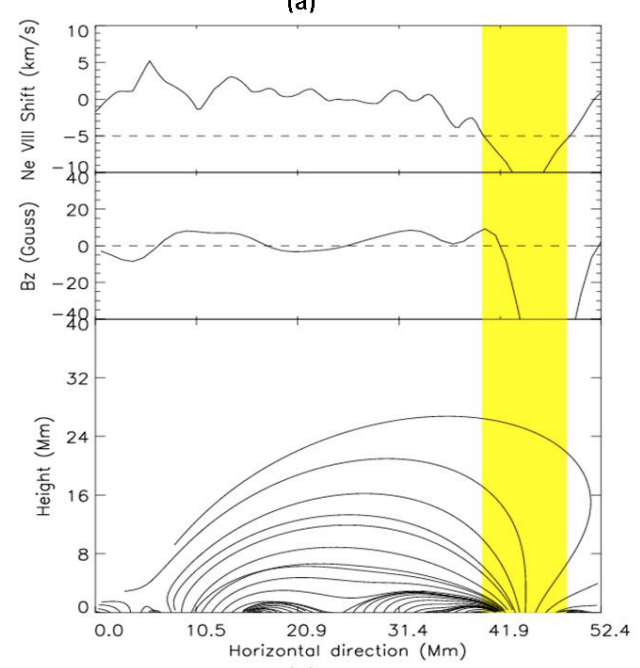

(c)

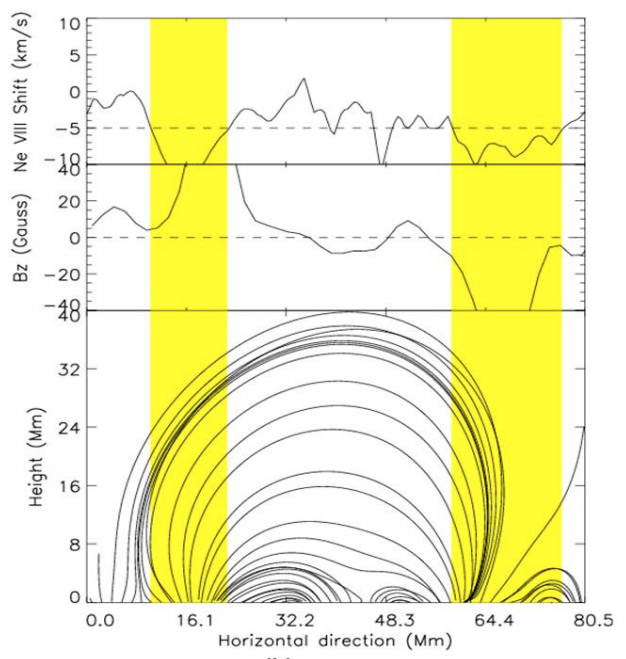

(b)

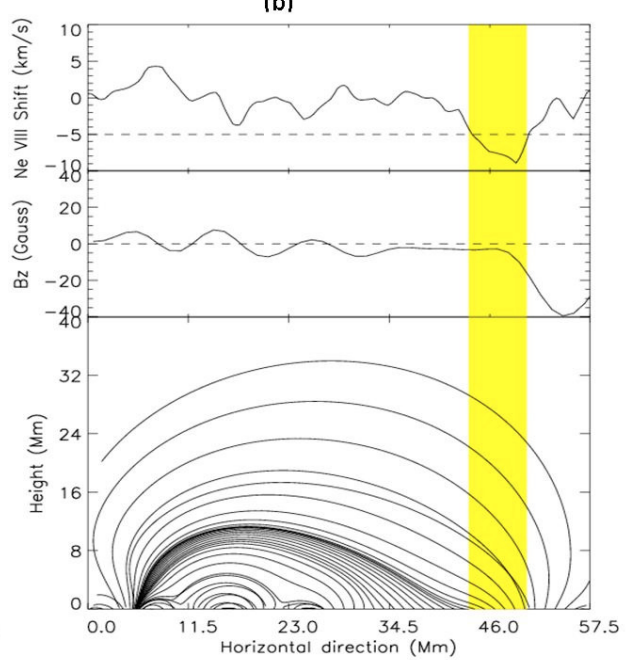

(d)
Fig. 4. For each subfigure, top panel: the Ne VIII Doppler shift; middle panel: the line-of-sight component of the observed photospheric magnetic field strength along the different cuts indicated by the yellow lines in Fig. 3; bottom panel: the magnetic field lines projected onto the vertical plane defined by a cut through the extrapolation box. The four subfigures correspond to the four cuts (in the sequence from left to right in Fig. 3) and are here indicated by letters a), b), c) and d), respectively. The yellow shaded stripes respectively mark those segments of the plane where the Ne VIII blue shift is observed to be strong (unsigned larger than $5 \mathrm{~km} \mathrm{~s}^{-1}$ ). a specific process, for example, most naturally from the bottom, or perhaps through side flows, or via reconnection with adjacent field lines of small neighboring loops, just like in the scenario suggested by Tu et al. (2005a,c) for the coronal hole funnels. After having entered the loop legs, the plasma flows up and may accumulate around the loop top, and then be released to the surrounding corona when the loop may transiently open through instability or reconnection.

Our present result revealing that most of the small funnels are not associated with strong outflow seems puzzling, especially if solar wind is assumed to flow out along open field lines, as suggested by $\mathrm{He}$ et al. (2007). One possible reason is that the outflow in quiet-Sun coronal funnels is an occasional phenomenon due to small-scale magnetic activity rather than a continuous one. In fact, the SUMER raster was done over $7.5 \mathrm{~h}$, while the MDI magnetogram reflects the magnetic activity for a short part of this period. Even though supergranulations have a lifetime of roughly a day $(>7.5 \mathrm{~h})$, the network magnetic field is believed to be constantly reshuffled $(>7.5 \mathrm{~h})$, which can produce outflow occasionally around the relatively unchanged network boundary. However, only those outflows occurring during an exposure time can be recorded by SUMER. The fact that one small funnel reveals considerable blue shift at its bottom (around the coordinates $x=220^{\prime \prime}$ and $y=75^{\prime \prime}$ in the $x-y$ plane) seems to support this conjecture. However, it must be recognized that the Ne VIII blue shift can perhaps not be used as a reliable tracer of solar wind outflow in the quiet Sun, although this assumption is considered to be appropriate for coronal holes. Since the magnetic structures in a coronal hole and the quiet Sun are quite different, it is likely that in a quiet-Sun region the location where prominent solar wind outflow starts is higher than the source of the Ne VIII emission, and significant outflow could perhaps be found only in the dopplergram of an ion with a higher formation temperature than that of the Ne VIII line. This prediction is consistent with the results of Tu et al. (2005a,b), who obtained a correlation height of $20.6 \mathrm{Mm}$ for Ne VIII in a coronal hole and a much lower correlation height of only $3.7 \mathrm{Mm}$ in a quiet-Sun region. This prediction is hard to be confirmed with the present data set, because there was no ion with a higher formation temperature than Ne VIII in the spectral window covered by this data. We suggest to employ the emission lines of EIS/HINODE to check whether prominent outflow can be found in dopplergrams of an ion formed at a higher temperature than Ne VIII in the quiet Sun.

Finally, we will roughly estimate the supply of mass to a coronal loop under the assumption that the deduced Doppler shift of the Ne VIII line represents the real outflow velocity of the neon ions, which are considered as markers of the proton 
flow. For a given loop leg we have, through the mass continuity equation, the relation:

$f=N_{\mathrm{e}} V A$,

where $f, N_{\mathrm{e}}, V$ and $A$ are the mass flux, electron density, outflow velocity and area of the loop cross section at the height of the Ne VIII emission, respectively.

We took for the electron density in the quiet transition region the value $N_{\mathrm{e}}=10^{9.64} \mathrm{~cm}^{-3}$ from Griffiths et al. (1999). Here, we consider case (b) of Fig. 4, for example. First we selected patches with a blue shift larger than $5 \mathrm{~km} \mathrm{~s}^{-1}$ around the two legs of the loop system on the Ne VIII dopplergram, and got a value of $376 \operatorname{arcsec}^{2}$ and $373 \operatorname{arcsec}^{2}$ for the two leg areas. These sizes are slightly larger than the size of a patch of the chromospheric network lane with a typical value of $\pi \times 10^{2} \operatorname{arcsec}^{2}=$ $314 \operatorname{arcsec}^{2}$, and thus can be used as estimates of the area of the loop cross section at the height of Ne VIII emission source. We then averaged the blue shifts in the two patches, and got average values of $6.92 \mathrm{~km} \mathrm{~s}^{-1}$ and $9.26 \mathrm{~km} \mathrm{~s}^{-1}$, which were used as the average outflow velocities in the two legs of the loop. Finally, we substituted these parameters into Eq. (1), and thus the mass supply rate or mass flux into the two legs of the coronal loop system can be estimated as being of the order of $10^{34} \mathrm{~s}^{-1}$.

Acknowledgements. We thank the anonymous referee for his/her careful reading of the paper and for the comments and suggestions. We also thank Dr. L.-D. Xia, C. Zhou and L. Zhao for their initial support of SUMER data analysis.

H. Tian, C.-Y. Tu, J.-S. He, and G.-Q Zhou are supported by the National Natural Science Foundation of China under contracts 40574078, 40336053 and 40436015, and by the Beijing Education Project XK100010404, as well as the foundation Major Project of National Basic Research contract 2006CB806305. H. Tian is also supported by China Scholarship Council for his stay in the MaxPlanck-Institut für Sonnensystemforschung in Germany.
The SUMER project is financially supported by DLR, CNES, NASA, and the ESA PRODEX programme (Swiss contribution). SUMER and MDI are instruments on board SOHO, an ESA and NASA mission. We thank the teams of SUMER and MDI for the spectroscopic and magnetic field data.

\section{References}

Aiouaz, T., \& Rast, M. P. 2006, ApJ, 647, L183

Aiouaz, T., Peter, H., \& Lemaire, P. 2005, A\&A, 435, 713

Brekke, P., Hassler, D. M., \& Wilhelm, K. 1997, Sol. Phys., 175, 349

Curdt, W., Landi, E., \& Feldman, U. 2004, A\&A,427,1045

Dammasch, I. E., Wilhelm, K., Curdt, W., \& Hassler, D. M. 1999, A\&A, 346, 285

Griffiths, N. W., Fisher, G. H., Woods, D. T., \& Siegmund,O. H. W. 1999, ApJ, 512,992

Gontikakis, C., Peter, H., \& Dara, H. C. 2003, A\&A,408,743

Hassler, D. M., Dammasch, I. E., Lemaire, P., et al. 1999, Science, 283, 810

He, J.-S., Tu, C.-Y., \& Marsch, E. 2007, A\&A, 468, 307

Mariska, J. T. 1988, ApJ, 334, 489

Marsch, E., Wiegelmann, T., \& Xia, L.-D. 2004, A\&A, 428, 629

McClymont, A. N., \& Craig, I. J. D. 1987, ApJ, 312, 402

Peter, H. 1999, ApJ, 516, 490

Peter, H. 2000, A\&A, 360,761

Peter, H., \& Judge, P. G. 1999, ApJ, 522, 1148

Seehafer, N. 1978, Sol. Phys., 58, 215

Spadaro, D., Antiochos, S. K., \& Mariska, J. T. 1991, ApJ 382, 338

Stucki, K., Solanki, S. K., Schühle, U., et al. 2000, A\&A, 363, 1145

Tu, C.-Y., Zhou, C., Marsch, E., et al. 2005a, Science, 308, 519

Tu, C.-Y., Zhou, C., Marsch, E., et al. 2005b, ApJ, 624, L133

Tu, C.-Y., Zhou, C., Marsch, E., et al. 2005c, Proceedings of SW 11-SOHO 16, Connecting Sun and Heliosphere, Whistler, Canada, 12-17 June 2005, ESA SP-592

Wilhelm, K., Dammasch, I. E., Marsch, E., \& Hassler D. M. 2000, A\&A, 353, 749

Xia, L. D., Marsch, E., \& Curdt, W. 2003, A\&A, 399, L5 